\title{
Interpolation of a regular subspace complementing the span of a radially singular function
}

\author{
by
}

Konstantin Zerulla (Karlsruhe)

\begin{abstract}
We analyze the interpolation of the sum of a subspace, consisting of regular functions, with the span of a function with $r^{\alpha}$-type singularity. In particular, we determine all interpolation parameters, for which the interpolation space of the subspace of regular functions is still a closed subspace. The main tool is here a result by Ivanov and Kalton on interpolation of subspaces. To apply it, we study the $K$-functional of the $r^{\alpha}$-singular function. It turns out that the $K$-functional possesses upper and lower bounds that have a common decay rate at zero.
\end{abstract}

1. Introduction. Many relevant problems in mathematics and physics demand for a thorough study of functions that have a radial singularity of $r^{\alpha}$ type. Important examples are elliptic boundary value problems on domains with irregular boundary, or interface problems for an elliptic operator (see [12, 10, 21, 3, 16, 8, 9] for instance). These functions furthermore play an important role in the analysis and numerical solution of Maxwell equations on homogeneous and heterogeneous domains with irregular boundary (see [8, 9, 7, 2] among others). The error in numerical approximations for problems involving singular functions of $r^{\alpha}$-type is also investigated in [4, 5, 6] for instance.

This paper is motivated by the regularity analysis of Maxwell equations in heterogeneous cuboids, which is in preparation. Indeed, Theorem 1.1 below is essential for the study of the behavior of the electric field near interior edges of a heterogeneous material, as the regularity of the electric field can be expressed by means of the first interpolation space in Theorem 1.1 .

We consider here the singular function

$$
\omega(r \cos \varphi, r \sin \varphi)=\chi(r) r^{\alpha} \psi(\varphi), \quad r \in[0,1], \varphi \in[0,2 \pi),
$$

2020 Mathematics Subject Classification: 46B70, 26A30, 46E35.

Key words and phrases: subspace interpolation, $r^{\alpha}$-type singularity, $K$-functional, Sobolev spaces, modulus of smoothness.

Received 21 June 2021; revised 1 August 2021.

Published online 7 March 2022. 
on the open unit disc $D$. The definition $(1)$ involves a smooth cut-off function $\chi:[0, \infty) \rightarrow[0,1]$ with $\chi=1$ on $[0,1 / 2]$ and support in $[0,3 / 4]$, a number $\alpha \in(0,1)$, and a piecewise $\mathrm{C}^{2}$-function $\psi:[0,2 \pi] \rightarrow \mathbb{R}$ with $\psi(0)=1$. For simplicity, we assume that $\psi$ is $\mathrm{C}^{2}$-regular on $[0, \pi / 2]$ and on $[\pi / 2,2 \pi]$. Other partitions of $[0,2 \pi]$ or restrictions to subintervals can be handled with similar arguments. For brevity, we always write $\omega(r, \varphi)$ instead of $\omega(r \cos \varphi, r \sin \varphi)$. The span of $\omega$ is denoted by

$$
V:=\operatorname{span}\{\omega\} .
$$

We next present the main result of this paper. The relevant notation is introduced in Section 2. In particular, $\mathrm{PH}^{s}(D)$ denotes the space of piecewise $\mathrm{H}^{s}$-regular functions on $D$ for $s>0$ (see (3)).

THEOREM 1.1. The identities

$$
\begin{array}{lll}
\left(\mathrm{PH}^{1}(D), \mathrm{PH}^{2}(D) \oplus V\right)_{\theta_{1}, 2}=\mathrm{PH}^{1+\theta_{1}}(D) \oplus V, & \theta_{1} \in(\alpha, 1), \\
\left(\mathrm{PH}^{1}(D), \mathrm{PH}^{2}(D) \oplus V\right)_{\theta_{2}, 2}=\mathrm{PH}^{1+\theta_{2}}(D), & \theta_{2} \in(0, \alpha),
\end{array}
$$

are valid. For the critical value $\alpha$, the space $\mathrm{PH}^{1+\alpha}(D)$ is not closed in $\left(\mathrm{PH}^{1}(D), \mathrm{PH}^{2}(D) \oplus V\right)_{\alpha, 2}$.

We point out that the statements in Theorem 1.1 are sharp. Note also that the space $\mathrm{PH}^{1+\theta_{1}}(D) \oplus V$ is equipped with the sum of the norms in $\mathrm{PH}^{1+\theta_{1}}(D)$ and $V$.

To the best of our knowledge, Theorem 1.1 is the first one to answer the question for which interpolation parameters $\theta$ the interpolation space $\left(\mathrm{PH}^{1}(D), \mathrm{PH}^{2}(D)\right)_{\theta, 2}=\mathrm{PH}^{1+\theta}(D)$ is a closed subspace of the interpolation space $\left(\mathrm{PH}^{1}(D), \mathrm{PH}^{2}(D) \oplus V\right)_{\theta, 2}$. This question has been investigated for other spaces in the literature:

Consider an interpolation couple $\left(X_{0}, X_{1}\right)$, and closed subspaces $Y_{0}$ in $X_{0}$ and $Y_{1}$ in $X_{1}$. Is the interpolation space $\left(Y_{0}, Y_{1}\right)_{\theta, p}$ still a closed subspace of $\left(X_{0}, X_{1}\right)_{\theta, p}$ ?

The issue is addressed in [17, Chapter 1, Problem 18.5], and [17, Chapter 1, Remark 11.4] implies that the answer is no, in general. In [22, Satz 5], Triebel gives an example of Hilbert spaces $H_{0}, H_{1}, H_{2}$ with $H_{1} \hookrightarrow H_{0}$, $H_{2} \subseteq H_{1}$ being closed with (arbitrary) finite codimension, and $\left(H_{0}, H_{2}\right)_{1 / 2,2}$ not closed in $\left(H_{0}, H_{1}\right)_{1 / 2,2}$. Wallstén [23] analyzes this issue for a codimension 1 subspace $M$ in $\mathrm{L}^{1}$, and interpolates with $\mathrm{L}^{\infty}$. Depending on the choice of $M$ and the interpolation parameter, the interpolation space between $M$ and $\mathrm{L}^{\infty}$ is or is not a closed subspace of the interpolation space between $\mathrm{L}^{1}$ and $\mathrm{L}^{\infty}$. Note that it can also happen that the non-closedness occurs for any interpolation parameter in $(0,1)$ (see [23]).

Ivanov and Kalton [13] studied Banach spaces $X_{0}, X_{1}$ and $Y_{0}$, with $Y_{0}$ being a closed subspace of codimension 1 in $X_{0}$, and $\left(X_{0}, X_{1}\right)$ an interpolation 
couple. They derived formulas for numbers $\sigma_{0} \leq \sigma_{1}$ such that $\left(Y_{0}, X_{1}\right)_{\theta, p}$ is a closed subspace of $\left(X_{0}, X_{1}\right)_{\theta, p}$ for $\theta \in\left(0, \sigma_{0}\right) \cup\left(\sigma_{1}, 1\right), p \in[1, \infty)$ (see [13, Theorem 2.1]). Note that some of the statements in [13] were earlier obtained in [18]. Theorem 2.1 from [13] is the essential tool in the proof of Theorem 1.1 in this paper. In [1], the findings of [13] are generalized. In particular, the closed subspace $Y_{0}$ is allowed to have arbitrary finite codimension in $X_{0}$.

The major difficulty in the proof of Theorem 1.1 is to obtain a sharp lower bound for the $K$-functional of the singular function $\omega$. To that end, we study the modulus of smoothness of $\omega$. It turns out that a subtle analysis of $\omega$ near zero is needed to provide estimates for the modulus of smoothness (see the proof of Lemma 3.1.

The paper is organized in the following way. In the next section, we fix some notation from interpolation theory, and we introduce the relevant (broken) Sobolev spaces of fractional order. In Section 3, we derive the crucial upper and lower estimates for the $K$-functional of the singular function $\omega$. Finally, we deduce Theorem 1.1 in Section 4 by combining our results from Section 3 with [13, Theorem 2.1].

As a byproduct of our preparations in Section 3, we also obtain a precise regularity statement for $\omega$ in terms of interpolation spaces (see Corollary 3.3.) To the best of our knowledge, only parts of the statement are available in the literature (see 4] for instance).

2. Analytical preliminaries. We first recall basic constructions from real interpolation theory via the $K$-method. Our presentation follows [19, Section 1.1]. Let $\left(X,\|\cdot\|_{X}\right)$ and $\left(Y,\|\cdot\|_{Y}\right)$ be real Banach spaces, which both embed into a common Hausdorff space. The $K$-functional is given by the formula

$$
K(t, z, X, Y):=\inf _{\substack{z=x+y \\ x \in X, y \in Y}}\left(\|x\|_{X}+t\|y\|_{Y}\right)
$$

for $z \in X+Y$ and $t>0$. It is used to define the real interpolation spaces

$$
(X, Y)_{\theta, p}:=\left\{z \in X+Y \mid\|z\|_{(X, Y)_{\theta, p}}^{p}:=\int_{0}^{\infty} t^{-1-\theta p} K(t, z, X, Y)^{p} \mathrm{~d} t<\infty\right\}
$$

for $\theta \in(0,1)$ and $p \in[1, \infty)$. The spaces

$$
\begin{aligned}
(X, Y)_{\theta} & :=\left\{z \in X+Y \mid \lim _{t \rightarrow 0} t^{-\theta} K(t, z, X, Y)=\lim _{t \rightarrow \infty} t^{-\theta} K(t, z, X, Y)=0\right\}, \\
(X, Y)_{\theta, \infty} & :=\left\{z \in X+Y \mid t \mapsto t^{-\theta} K(t, z, X, Y) \in \mathrm{L}^{\infty}(0, \infty)\right\}
\end{aligned}
$$

also arise in this paper. Both are complete with respect to the norm

$$
\|z\|_{(X, Y)_{\theta, \infty}}:=\left\|t^{-\theta} K(t, z, X, Y)\right\|_{\mathrm{L}^{\infty}(0, \infty)}, \quad z \in(X, Y)_{\theta, \infty} .
$$


We next recall the definition of fractional order Sobolev spaces. Let $O \subseteq \mathbb{R}^{2}$ be a bounded domain with compact Lipschitz boundary. We denote the standard Sobolev space of order $k \in \mathbb{N}$ by $\mathrm{H}^{k}(O)$, and set

$$
\mathrm{H}^{s}(O):=\left(\mathrm{L}^{2}(O), \mathrm{H}^{2}(O)\right)_{s / 2,2}, \quad s \in(0,2) .
$$

Fractional Sobolev spaces can also be defined by means of weighted difference quotients (see [20, Section 2.3.8] for instance). Note that both definitions are equivalent in the current setting. (This well known fact is for instance verified by combining the main theorem in [15, Section 1] with [11, Corollary 6.8].)

Having the choice of the function $\psi$ in (1) in mind, we introduce the open disc segments

$$
\begin{aligned}
& D_{1}:=\{(r \cos \varphi, r \sin \varphi) \mid r \in(0,1), \varphi \in(0, \pi / 2)\}, \\
& D_{2}:=\{(r \cos \varphi, r \sin \varphi) \mid r \in(0,1), \varphi \in(\pi / 2,2 \pi)\},
\end{aligned}
$$

using polar coordinates $(r, \varphi)$ on $D$. To study functions that are only regular on $D_{1}$ and $D_{2}$, but not on $D$, we use the broken fractional order Sobolev spaces

$$
\mathrm{PH}^{s}(D):=\left\{f \in \mathrm{L}^{2}(D)|f|_{D_{i}} \in \mathrm{H}^{s}\left(D_{i}\right), i \in\{1,2\}\right\}, \quad s \in(0,2],
$$

which are complete with respect to the norm

$$
\|f\|_{\mathrm{PH}^{s}(D)}:=\left(\sum_{i=1}^{2}\left\|\left.f\right|_{D_{i}}\right\|_{\mathrm{H}^{s}\left(D_{i}\right)}^{2}\right)^{1 / 2}, \quad f \in \mathrm{PH}^{s}(D) .
$$

We also note the interpolation property

$$
\left(\mathrm{L}^{2}(D), \mathrm{PH}^{2}(D)\right)_{s / 2,2}=\mathrm{PH}^{s}(D), \quad s \in(0,2) .
$$

An essential part of the proof for Theorem 1.1 is the derivation of sharp upper and lower estimates for the functional $K\left(\cdot, \omega, \mathrm{L}^{2}(D), \mathrm{PH}^{2}(D)\right.$ ) (see Lemmas 3.1 and 3.2. By sharp we mean that the upper and lower bounds have the same decay rate near zero. To obtain the inequalities, it is useful to analyze the second modulus of smoothness for the singular function $\omega$ on an appropriately chosen open subset $D_{0}$ of $D_{1}$. To define the second modulus of smoothness, we use the set

$$
D_{0}(h)=\left\{v \in D_{0} \mid v+t h \in D_{0} \text { for all } 0 \leq t \leq 1\right\}
$$

for $h \in \mathbb{R}^{2}$. Denoting the characteristic function of a set $O \subseteq \mathbb{R}^{2}$ by $\mathbb{1}_{O}$, the second modulus of smoothness of $\omega$ on $D_{0}$ is defined as

$$
m_{2}(t, \omega):=\sup _{0<|h| \leq t}\left\|\mathbb{1}_{D_{0}(2 h)}(\omega-2 \omega(\cdot+h)+\omega(\cdot+2 h))\right\|_{L^{2}\left(D_{0}\right)},
$$

for $t>0$ (see [14, Section 1] for instance). [14, Lemma 1] and the definition 
of the $K$-functional then provide the inequality

$$
\begin{aligned}
K\left(t^{2}, \omega, \mathrm{L}^{2}\left(D_{0}\right), \mathrm{H}^{2}\left(D_{0}\right)\right) & \\
& \geq \inf _{g \in \mathrm{H}^{2}\left(D_{0}\right)}\left(\|\omega-g\|_{\mathrm{L}^{2}\left(D_{0}\right)}+t^{2} \sup _{k_{1}+k_{2}=2}\left\|\partial_{x}^{k_{1}} \partial_{y}^{k_{2}} g\right\|_{\mathrm{L}^{2}\left(D_{0}\right)}\right) \\
& \geq C m_{2}(t, \omega), \quad t>0,
\end{aligned}
$$

with a uniform constant $C>0$. We then infer the useful estimate

$$
\begin{aligned}
K\left(t^{2}, \omega, \mathrm{L}^{2}(D), \mathrm{PH}^{2}(D)\right) & =\inf _{\substack{\omega=f+g \\
f \in \mathrm{L}^{2}(D), g \in \mathrm{PH}^{2}(D)}}\left(\|f\|_{\mathrm{L}^{2}(D)}+t^{2}\|g\|_{\mathrm{PH}^{2}(D)}\right) \\
& \inf _{\substack{\omega=\tilde{f}+\tilde{g} \\
\tilde{f} \in \mathrm{L}^{2}\left(D_{0}\right), \tilde{g} \in \mathrm{H}^{2}\left(D_{0}\right)}}\left(\|\tilde{f}\|_{\mathrm{L}^{2}\left(D_{0}\right)}+t^{2}\|\tilde{g}\|_{\mathrm{H}^{2}\left(D_{0}\right)}\right) \\
& =K\left(t^{2}, \omega, \mathrm{L}^{2}\left(D_{0}\right), \mathrm{H}^{2}\left(D_{0}\right)\right) \geq C m_{2}(t, \omega)
\end{aligned}
$$

for $t>0$, which comes into play in the proof of Lemma 3.1 .

3. Estimates for the $K$-functional. In this section, we derive upper and lower estimates for the $K$-functional of the singular function $\omega$ from (1). The inequalities are crucial for the proof of Theorem 1.1 in Section 4. In particular, it is important to have upper and lower bounds for the $K$-functional that have the same decay rate near zero.

In the next lemma, we start with the lower estimate.

LEMma 3.1. We have

$$
K\left(t^{2}, \omega, \mathrm{L}^{2}(D), \mathrm{PH}^{2}(D)\right) \geq C_{l} t^{\alpha+1}, \quad t \in(0,1],
$$

with a uniform constant $C_{l}=C_{l}(\omega)>0$.

Proof. 1) We consider the problem in cartesian coordinates $(x, y)$ on the open subset

$$
D_{0}:=\left\{(x, y) \mid 0<x^{2}+y^{2}<1 / 16,0<y<x\right\}
$$

of $D_{1}$. Note that the cut-off function $\chi$ from (1) is then equal to 1 on $D_{0}$. As a result, $\omega$ has the representation

$$
\omega(x, y)=\psi(\arctan (y / x))|(x, y)|^{\alpha}, \quad(x, y) \in D_{0} .
$$

On $D_{0}$, we then calculate

$$
\begin{aligned}
\partial_{x} \omega(x, y)= & -y \psi^{\prime}(\arctan (y / x))|(x, y)|^{\alpha-2}+\alpha x \psi(\arctan (y / x))|(x, y)|^{\alpha-2}, \\
\partial_{x}^{2} \omega(x, y)= & \left(y^{2} \psi^{\prime \prime}(\arctan (y / x))-2(\alpha-1) y x \psi^{\prime}(\arctan (y / x))\right. \\
& \left.+\alpha(\alpha-2) x^{2} \psi(\arctan (y / x))\right)|(x, y)|^{\alpha-4} \\
& +\alpha \psi(\arctan (y / x))|(x, y)|^{\alpha-2} .
\end{aligned}
$$

We next derive a lower estimate for $-\partial_{x}^{2} \omega$ on an appropriate part of $D_{0}$. For convenience, we denote the piecewise $\mathrm{C}^{2}$-norm of $\psi$ by $\|\psi\|_{\mathrm{C}^{2}}$ (the supre- 
mum of the $\mathrm{C}^{2}$-norms on $D_{1}$ and $D_{2}$ ). Recall that $\psi(0)=1$ (see Section 1 ). By continuity, there is a $\delta \in(0, \pi / 2)$ such that $\psi(\varphi) \geq 1 / 2$ for all $\varphi \in[0, \delta]$. Let furthermore $x \in\left(0, \frac{1}{4 \sqrt{10}}\right)$ and $0<y \leq \gamma x$ with

$$
\gamma:=\min \left\{\tan \delta, \frac{(1-\alpha) \alpha}{12\|\psi\|_{\mathrm{C}^{2}}}\right\}
$$

Note that $\gamma<1$, as $\psi(0)=1$ and $\alpha<1$. The choice of $\gamma$ in particular implies that $\arctan (y / x) \in(0, \delta]$. In view of the assumption $\alpha \in(0,1)$, we get

(6) $\left(\alpha(2-\alpha) x^{2} \psi(\arctan (y / x))-y^{2} \psi^{\prime \prime}(\arctan (y / x))\right.$

$$
\begin{aligned}
& \left.-2(1-\alpha) y x \psi^{\prime}(\arctan (y / x))\right)|(x, y)|^{\alpha-4} \\
\geq & \left(\frac{1-\alpha-2 \gamma}{2-\alpha} \alpha(2-\alpha) x^{2} \psi(\arctan (y / x))+\frac{1+2 \gamma}{2-\alpha} \alpha(2-\alpha) x^{2} \psi(\arctan (y / x))\right. \\
& \left.-y^{2}\|\psi\|_{\mathrm{C}^{2}}-2 y x\|\psi\|_{\mathrm{C}^{2}}\right)|(x, y)|^{\alpha-4} \\
\geq & \left(\frac{1}{2}(1-\alpha-2 \gamma) \alpha x^{2}+(1+2 \gamma) \alpha x^{2} \psi(\arctan (y / x))\right. \\
& \left.-3 \gamma x^{2}\|\psi\|_{\mathrm{C}^{2}}\right)|(x, y)|^{\alpha-4} .
\end{aligned}
$$

We next use $\gamma \leq \frac{(1-\alpha) \alpha}{12\|\psi\|_{\mathrm{C}^{2}}}<\frac{1-\alpha}{4}$ to deduce that

$$
\begin{aligned}
& \left(\frac{1}{2}(1-\alpha-2 \gamma) \alpha x^{2}+(1+2 \gamma) \alpha x^{2} \psi(\arctan (y / x))-3 \gamma x^{2}\|\psi\|_{\mathrm{C}^{2}}\right)|(x, y)|^{\alpha-4} \\
& \geq\left(\frac{1}{4}(1-\alpha) \alpha x^{2}+(1+2 \gamma) \alpha x^{2} \psi(\arctan (y / x))-3 \gamma x^{2}\|\psi\|_{\mathrm{C}^{2}}\right)|(x, y)|^{\alpha-4} \\
& \geq(1+2 \gamma) \alpha x^{2} \psi(\arctan (y / x))|(x, y)|^{\alpha-4} .
\end{aligned}
$$

Combining (6)-(7) and using again the relation $y \leq \gamma x$, we infer that

$$
\begin{aligned}
& -\partial_{x}^{2} \omega(x, y) \\
& \geq-\alpha \psi(\arctan (y / x))|(x, y)|^{\alpha-2}+(1+2 \gamma) \alpha x^{2} \psi(\arctan (y / x))|(x, y)|^{\alpha-4} \\
& =\alpha\left(-\left(x^{2}+y^{2}\right)+(1+2 \gamma) x^{2}\right) \psi(\arctan (y / x))|(x, y)|^{\alpha-4} \\
& \geq \alpha(-(1+\gamma)+1+2 \gamma) x^{2} \psi(\arctan (y / x))|(x, y)|^{\alpha-4} \\
& =\alpha \gamma x^{2} \psi(\arctan (y / x))|(x, y)|^{\alpha-4} .
\end{aligned}
$$

2) We now bound the second modulus of smoothness $m_{2}(\cdot, \omega)$ for $\omega$ on $D_{0}$ from below (see (4)). To that end, we choose $t<\frac{1}{12 \sqrt{10}}$ and $h=\left(h_{1}, 0\right)$ with $h_{1}=t$ in (4). Combining the choice of $h_{1}$ and $\gamma<1$, we obtain

$$
\begin{aligned}
m_{2}(t, \omega)^{2} & \geq \int_{0}^{h_{1}} \int_{0}^{\gamma x}\left(\omega(x, y)-2 \omega\left(x+h_{1}, y\right)+\omega\left(x+2 h_{1}, y\right)\right)^{2} \mathrm{~d} y \mathrm{~d} x \\
& =\int_{0}^{h_{1}} \int_{0}^{\gamma x}\left(\int_{0}^{h_{1}}-\partial_{x} \omega(x+s, y)+\partial_{x} \omega\left(x+h_{1}+s, y\right) \mathrm{d} s\right)^{2} \mathrm{~d} y \mathrm{~d} x \\
& =\int_{0}^{h_{1}} \int_{0}^{\gamma x}\left(\int_{0}^{h_{1}} \int_{0}^{h_{1}} \partial_{x}^{2} \omega(x+s+\tau, y) \mathrm{d} \tau \mathrm{d} s\right)^{2} \mathrm{~d} y \mathrm{~d} x .
\end{aligned}
$$


Inserting now also (8), we infer that

$$
\begin{aligned}
m_{2}(t, \omega)^{2} \geq \int_{0}^{h_{1}} \int_{0}^{\gamma x}\left(\int_{0}^{h_{1}} \int_{0}^{h_{1}} \alpha \gamma(x+s+\tau)^{2} \psi\left(\arctan \left(\frac{y}{x+s+\tau}\right)\right)\right. \\
\left.\cdot|(x+s+\tau, y)|^{\alpha-4} \mathrm{~d} \tau \mathrm{d} s\right)^{2} \mathrm{~d} y \mathrm{~d} x .
\end{aligned}
$$

Taking also into account the facts that $\psi\left(\arctan \left(\frac{y}{x+s+\tau}\right)\right) \geq \frac{1}{2}$ for $x, s, \tau \in$ $\left(0, h_{1}\right)$ and $y<\gamma x$, we arrive at

$$
\begin{aligned}
& m_{2}(t, \omega)^{2} \\
& \quad \geq \int_{0}^{h_{1}} \int_{0}^{\gamma x}\left(\int_{0}^{h_{1}} \int_{0}^{h_{1}} \frac{\alpha}{2} \gamma(x+s+\tau)^{2}(1+\gamma)^{\alpha-4}(x+s+\tau)^{\alpha-4} \mathrm{~d} \tau \mathrm{d} s\right)^{2} \mathrm{~d} y \mathrm{~d} x \\
& \quad=\int_{0}^{h_{1}} \int_{0}^{\gamma x}\left(\int_{0}^{h_{1}} \frac{\alpha}{2} \gamma \frac{(1+\gamma)^{\alpha-4}}{1-\alpha}\left((x+s)^{\alpha-1}-\left(x+s+h_{1}\right)^{\alpha-1}\right) \mathrm{d} s\right)^{2} \mathrm{~d} y \mathrm{~d} x \\
& \geq \int_{0}^{h_{1}} \int_{0}^{\gamma x}\left(\int_{0}^{h_{1}} \frac{\alpha}{2} \gamma \frac{(1+\gamma)^{\alpha-4}}{1-\alpha}\left(1-\left(\frac{3}{2}\right)^{\alpha-1}\right)(x+s)^{\alpha-1} \mathrm{~d} s\right)^{2} \mathrm{~d} y \mathrm{~d} x \\
& \geq \int_{0}^{h_{1}} \int_{0}^{\gamma x}\left(\int_{0}^{h_{1}} \frac{\alpha}{2} \gamma \frac{(1+\gamma)^{\alpha-4}}{1-\alpha}\left(1-\left(\frac{3}{2}\right)^{\alpha-1}\right) 2^{\alpha-1} h_{1}^{\alpha-1} \mathrm{~d} s\right)^{2} \mathrm{~d} y \mathrm{~d} x \\
& =\frac{\alpha^{2}}{4^{1-\alpha}} \gamma^{3} \frac{(1+\gamma)^{2 \alpha-8}}{8(1-\alpha)^{2}}\left(1-\left(\frac{3}{2}\right)^{\alpha-1}\right)^{2} h_{1}^{2 \alpha+2}=: C_{1} h_{1}^{2 \alpha+2} .
\end{aligned}
$$

In view of $(5)$, we hence get

$$
K\left(t^{2}, \omega, \mathrm{L}^{2}(D), \mathrm{PH}^{2}(D)\right) \geq C \sqrt{C_{1}} t^{\alpha+1}, \quad 0<t<\frac{1}{12 \sqrt{10}}=: t_{0} .
$$

The monotonicity of the $K$-functional furthermore implies

$$
K\left(t^{2}, \omega, \mathrm{L}^{2}(D), \mathrm{PH}^{2}(D)\right) \geq K\left(t_{0}^{2}, \omega, \mathrm{L}^{2}(D), \mathrm{PH}^{2}(D)\right)=: C_{2} \geq C_{2} t^{\alpha+1}
$$

for $t \in\left[t_{0}, 1\right]$. Altogether, we arrive at the desired statement.

The remaining upper estimate for the $K$-functional essentially follows from a modification of the arguments in the proofs of [5], Theorem 2.3] and [6. Theorem 2.5]. For the sake of a clear presentation, however, we elaborate the proof.

LEMMA 3.2. There is a uniform constant $C_{u}=C_{u}(\omega)>0$ such that

$$
K\left(t^{2}, \omega, \mathrm{L}^{2}(D), \mathrm{PH}^{2}(D)\right) \leq C_{u} t^{\alpha+1}, \quad t \in(0,1] .
$$

Proof. 1) Throughout the proof, $C=C(\omega)>0$ is a constant that is allowed to change from line to line. Let $\delta \in(0,1)$ be a fixed number, to be determined later. Let furthermore $\chi_{\delta}:[0,1] \rightarrow[0,1]$ be a smooth cut-off 
function with $\chi_{\delta}=1$ on $[0, \delta / 2]$, with support in $[0, \delta],\left\|\chi_{\delta}^{\prime}\right\|_{\infty} \leq C / \delta$, and $\left\|\chi_{\delta}^{\prime \prime}\right\|_{\infty} \leq C / \delta^{2}$. We then write $\omega$ as the sum

$$
\omega(r, \varphi)=\chi_{\delta}(r) \omega(r, \varphi)+\left(1-\chi_{\delta}(r)\right) \omega(r, \varphi)=: v_{1}(r, \varphi)+v_{2}(r, \varphi)
$$

for $r \in[0,1]$ and $\varphi \in[0,2 \pi)$. By construction, $v_{2}$ is piecewise $\mathrm{C}^{2}$-regular on the partition $\bigcup_{i=1}^{2} \overline{D_{i}}$. As a result,

$$
\begin{aligned}
K\left(t^{2}, \omega, \mathrm{L}^{2}(D), \mathrm{PH}^{2}(D)\right) & =\inf _{\substack{\omega=\tilde{v}_{1}+\tilde{v}_{2} \\
\tilde{v}_{1} \in \mathrm{L}^{2}(D), \tilde{v}_{2} \in \mathrm{PH}^{2}(D)}}\left(\left\|\tilde{v}_{1}\right\|_{\mathrm{L}^{2}(D)}+t^{2}\left\|\tilde{v}_{2}\right\|_{\mathrm{PH}^{2}(D)}\right) \\
& \leq\left\|v_{1}\right\|_{\mathrm{L}^{2}(D)}+t^{2}\left\|v_{2}\right\|_{\mathrm{PH}^{2}(D)} .
\end{aligned}
$$

We next bound the quantities on the right hand side of (9) separately. Note that we only focus on the disc segment $D_{1}$; the remaining one can be handled in the same way, by symmetry.

2) Recall the definition

$$
\omega(r, \varphi)=\chi(r) r^{\alpha} \psi(\varphi)
$$

of $\omega$ in (1). Since the cut-off functions $\chi$ and $\chi_{\delta}$ are bounded by 1 , we have

$$
\left\|v_{1}\right\|_{L^{2}\left(D_{1}\right)}^{2} \leq C\|\psi\|_{\infty}^{2} \int_{0}^{\delta} r^{2 \alpha+1} \mathrm{~d} r \leq C \delta^{2 \alpha+2} .
$$

3) Similar to 2), we first bound the $L^{2}$-norm of $v_{2}$ :

$$
\left\|v_{2}\right\|_{\mathrm{L}^{2}\left(D_{1}\right)}^{2} \leq C \delta^{2 \alpha-2} .
$$

To estimate the $\mathrm{H}^{2}$-norm of $v_{2}$ on $D_{1}$, we note that

$$
\begin{array}{r}
\left\|v_{2}\right\|_{\mathrm{H}^{2}\left(D_{1}\right)}^{2} \leq C\left(\left\|v_{2}\right\|_{\mathrm{L}^{2}\left(D_{1}\right)}^{2}+\int_{\delta / 2}^{1} \int_{0}^{\pi / 2}\left(r\left|\partial_{r}^{2} v_{2}\right|^{2}+\frac{1}{r}\left|\partial_{r} \partial_{\varphi} v_{2}\right|^{2}+\frac{1}{r^{3}}\left|\partial_{\varphi} v_{2}\right|^{2}\right.\right. \\
\left.\left.+\frac{1}{r}\left|\partial_{r} v_{2}\right|^{2}+\frac{1}{r^{3}}\left|\partial_{\varphi}^{2} v_{2}\right|^{2}\right) \mathrm{~d} \varphi \mathrm{d} r\right),
\end{array}
$$

which follows from the representation of all first and second order derivatives in polar coordinates, and the location of the support of $\chi_{\delta}$. The expressions on the right hand side of 12 are given by

$$
\begin{aligned}
\partial_{r} v_{2}= & \left(-\chi_{\delta}^{\prime} \chi r^{\alpha}+\left(1-\chi_{\delta}\right)\left(\chi^{\prime} r^{\alpha}+\alpha \chi r^{\alpha-1}\right)\right) \psi, \\
\partial_{r}^{2} v_{2}= & \left(\left(-\chi_{\delta}^{\prime \prime} \chi-2 \chi_{\delta}^{\prime} \chi^{\prime}+\left(1-\chi_{\delta}\right) \chi^{\prime \prime}\right) r^{\alpha}+2 \alpha\left(\left(1-\chi_{\delta}\right) \chi^{\prime}-\chi_{\delta}^{\prime} \chi\right) r^{\alpha-1}\right. \\
& \left.+\alpha(\alpha-1)\left(1-\chi_{\delta}\right) \chi r^{\alpha-2}\right) \psi \\
\partial_{\varphi} v_{2}= & \left(1-\chi_{\delta}\right) \chi r^{\alpha} \psi^{\prime} \\
\partial_{\varphi}^{2} v_{2}= & \left(1-\chi_{\delta}\right) \chi r^{\alpha} \psi^{\prime \prime} \\
\partial_{\varphi} \partial_{r} v_{2}= & \left(-\chi_{\delta}^{\prime} \chi r^{\alpha}+\left(1-\chi_{\delta}\right)\left(\chi^{\prime} r^{\alpha}+\alpha \chi r^{\alpha-1}\right)\right) \psi^{\prime} .
\end{aligned}
$$


Combining the choice of $\chi_{\delta}, 111$ and (12), we then obtain the estimates

$$
\begin{aligned}
\left\|v_{2}\right\|_{\mathrm{H}^{2}\left(D_{1}\right)}^{2} \leq C\left(\left\|v_{2}\right\|_{\mathrm{L}^{2}\left(D_{1}\right)}^{2}+\int_{\delta / 2}^{\delta}\left(\left|\chi_{\delta}^{\prime \prime}\right|^{2} r^{2 \alpha+1}+\left|\chi_{\delta}^{\prime}\right|^{2} r^{2 \alpha-1}\right)\|\psi\|_{\mathrm{C}^{2}} \mathrm{~d} r\right. & \left.+\int_{\delta / 2}^{1} r^{2 \alpha-3} \mathrm{~d} r\|\psi\|_{\mathrm{C}^{2}}\right) \\
& \leq C\left(\delta^{2 \alpha-2}+\int_{\delta / 2}^{\delta}\left(\frac{1}{\delta^{4}} r^{2 \alpha+1}+\frac{1}{\delta^{2}} r^{2 \alpha-1}\right) \mathrm{d} r\right) \leq C \delta^{2 \alpha-2} .
\end{aligned}
$$

By symmetry, an analogous inequality is true on $D_{2}$. As a result,

$$
\left\|v_{2}\right\|_{\mathrm{PH}^{2}(D)} \leq C \delta^{\alpha-1} .
$$

4) In view of (9), 10) and (13), we arrive at

$$
K\left(t^{2}, \omega, \mathrm{L}^{2}(D), \mathrm{PH}^{2}(D)\right) \leq C\left(\delta^{\alpha+1}+t^{2} \delta^{\alpha-1}\right) .
$$

The asserted statement follows by choosing $\delta=t$.

Combining Lemmas 3.1 and 3.2 , we can directly derive the following regularity statement for $\omega$ in terms of interpolation spaces. The first part of the statement is well known (see 4] for instance).

Corollary 3.3. Let $p \in[1, \infty)$ and $\theta \in\left(0, \frac{1+\alpha}{2}\right)$. The function $\omega$ is an element of the space

$$
\left(\mathrm{L}^{2}(D), \mathrm{PH}^{2}(D)\right)_{\theta, p} \cap\left(\mathrm{L}^{2}(D), \mathrm{PH}^{2}(D)\right)_{(1+\alpha) / 2, \infty},
$$

but not of the (continuous) interpolation space $\left(\mathrm{L}^{2}(D), \mathrm{PH}^{2}(D)\right)_{(1+\alpha) / 2}$.

Proof. The first statement is a direct consequence of Lemma 3.2 and the embeddings

$$
\left(\mathrm{L}^{2}(D), \mathrm{PH}^{2}(D)\right)_{(1+\alpha) / 2, \infty} \subset\left(\mathrm{L}^{2}(D), \mathrm{PH}^{2}(D)\right)_{\theta, 1} \subset\left(\mathrm{L}^{2}(D), \mathrm{PH}^{2}(D)\right)_{\theta, p}
$$

(see for instance [19, Propositions 1.3 and 1.4]). The last claim follows from Lemma 3.1 .

4. Proof of Theorem 1.1. The essential ingredients of the proof are an application of [13, Theorem 2.1], and the estimates for the $K$-functional of $\omega$ from Lemmas 3.1 and 3.2 .

To transform our problem into the setting of Ivanov and Kalton, we introduce the linear functional

$$
\Phi(v+\lambda \omega):=\lambda, \quad v \in \mathrm{PH}^{2}(D), \lambda \in \mathbb{R},
$$

on the space $\mathrm{PH}^{2}(D) \oplus V$. The latter is equipped with the norm

$$
\|v+\lambda \omega\|^{\prime}:=|\lambda|+\|v\|_{\mathrm{PH}^{2}(D)}, \quad v \in \mathrm{PH}^{2}(D), \lambda \in \mathbb{R} .
$$


The kernel of $\Phi$ then coincides with $\mathrm{PH}^{2}(D)$, and $\Phi$ is bounded. Following [13, Section 2], we also define the quantities

$$
\begin{aligned}
\|\Phi\|_{t} & :=\sup \left\{|\Phi(f)| \mid f \in \mathrm{PH}^{2}(D) \oplus V \text { with } t\|f\|_{\mathrm{L}^{2}(D)}+\|f\|^{\prime} \leq 1\right\}, \\
\sigma_{0} & :=\lim _{\tau \rightarrow \infty} \inf _{0<\tau t \leq 1} \frac{1}{\log \tau} \log \frac{K(\tau t, \Phi)}{K(t, \Phi)}, \\
\sigma_{1} & :=\lim _{\tau \rightarrow \infty} \sup _{0<\tau t \leq 1} \frac{1}{\log \tau} \log \frac{K(\tau t, \Phi)}{K(t, \Phi)},
\end{aligned}
$$

involving the function

$$
K(t, \Phi):=K\left(t, \Phi,\left(\mathrm{PH}^{2}(D) \oplus V\right)^{*}, \mathrm{~L}^{2}(D)^{*}\right), \quad t>0 .
$$

(Here $W^{*}$ denotes the dual space of $W$.) The reasoning in [13, proof of Proposition 3.2] then gives rise to the useful formulas

$$
\sigma_{0}=\lim _{\tau \rightarrow \infty} \inf _{s \geq 1} \frac{1}{\log \tau} \log \frac{\|\Phi\|_{s}}{\|\Phi\|_{s \tau}}, \quad \sigma_{1}=\lim _{\tau \rightarrow \infty} \sup _{s \geq 1} \frac{1}{\log \tau} \log \frac{\|\Phi\|_{s}}{\|\Phi\|_{s \tau}} .
$$

We next determine $\sigma_{0}$ and $\sigma_{1}$ in terms of the exponent $\alpha$.

LEMma 4.1. We have

$$
\sigma_{0}=\sigma_{1}=\frac{1-\alpha}{2}
$$

Proof. 1) For convenience, we write $K(\cdot, \omega)$ instead of $K\left(\cdot, \omega, \mathrm{L}^{2}(D)\right.$, $\left.\mathrm{PH}^{2}(D)\right)$. Let $t>0$, and $f=v+\lambda \omega$ in $\mathrm{PH}^{2}(D) \oplus V$ with $t\|f\|_{\mathrm{L}^{2}(D)}+\|f\|^{\prime} \leq 1$. If $\lambda$ is zero, then clearly

$$
|\Phi(f)|=0 \leq \frac{1}{1+t K(1 / t, \omega)} .
$$

The next goal is to establish the same estimate for nonzero real $\lambda$. By definition of the norm $\|\cdot\|^{\prime}$, we infer the estimates

$$
\begin{aligned}
1 & \geq t|\lambda|\left(\left\|\frac{1}{\lambda} v+\omega\right\|_{\mathrm{L}^{2}(D)}+\frac{1}{t}\left\|\frac{1}{\lambda} v\right\|_{\mathrm{PH}^{2}(D)}\right)+|\lambda| \\
& \geq t|\lambda| K(1 / t, \omega)+|\lambda|=(1+t K(1 / t, \omega))|\Phi(f)| .
\end{aligned}
$$

Taking now the supremum with respect to all $f$ in $\mathrm{PH}^{2}(D) \oplus V$ with $t\|f\|_{\mathrm{L}^{2}(D)}+\|f\|^{\prime} \leq 1$, we conclude that

$$
\|\Phi\|_{t} \leq \frac{1}{1+t K(1 / t, \omega)} .
$$

Next, we derive a similar lower bound for $\|\Phi\|_{t}$. To that end, let $z \in$ $\mathrm{PH}^{2}(D)$ with

$$
K(1 / t, \omega)+1 / t \geq\|z+\omega\|_{\mathrm{L}^{2}(D)}+\frac{1}{t}\|z\|_{\mathrm{PH}^{2}(D)} .
$$

We then put $v:=\|\Phi\|_{t} z \in \mathrm{PH}^{2}(D)$. Note that

$$
t\|v+\| \Phi\left\|_{t} \omega\right\|_{\mathrm{L}^{2}(D)}+\|\Phi\|_{t}+\|v\|_{\mathrm{PH}^{2}(D)} \geq 1,
$$


in view of the definition of $\|\Phi\|_{t}$. Combining (16) and (17), we obtain

$$
\begin{aligned}
1 & \geq \frac{\left\|\frac{1}{\|\Phi\|_{t}} v+\omega\right\|_{\mathrm{L}^{2}(D)}+\frac{1}{t}\left\|_{\frac{1}{\|\Phi\|_{t}}} v\right\|_{\mathrm{PH}^{2}(D)}}{K(1 / t, \omega)+1 / t} \\
& =\frac{\frac{1}{t\|\Phi\|_{t}}\left(t\|v+\| \Phi\left\|_{t} \omega\right\|_{\mathrm{L}^{2}(D)}+\|v\|_{\mathrm{PH}^{2}(D)}\right)}{K(1 / t, \omega)+1 / t} \\
& =\frac{\frac{1}{t\|\Phi\|_{t}}\left(t\|v+\| \Phi\left\|_{t} \omega\right\|_{\mathrm{L}^{2}(D)}+\|\Phi\|_{t}+\|v\|_{\mathrm{PH}^{2}(D)}-\|\Phi\|_{t}\right)}{K(1 / t, \omega)+1 / t} \\
& \geq \frac{1}{K(1 / t, \omega)+1 / t}\left(1-\|\Phi\|_{t}\right)
\end{aligned}
$$

Simple algebraic manipulations then lead to the desired inequality

$$
\|\Phi\|_{t} \geq \frac{1}{t K(1 / t, \omega)+2} .
$$

2) To bound $\sigma_{0}$ from below, we plug (15) and (18) into (14), which yields

$$
\sigma_{0}=\lim _{\tau \rightarrow \infty} \inf _{s \geq 1} \frac{1}{\log \tau} \log \frac{\|\Phi\|_{s}}{\|\Phi\|_{s \tau}} \geq \lim _{\tau \rightarrow \infty} \inf _{s \geq 1} \frac{1}{\log \tau} \log \left(\frac{s \tau K\left(\frac{1}{s \tau}, \omega\right)+1}{s K\left(\frac{1}{s}, \omega\right)+2}\right) .
$$

Taking now also Lemmas 3.1 and 3.2 into account, we arrive at

$$
\sigma_{0} \geq \lim _{\tau \rightarrow \infty} \inf _{s \geq 1} \frac{1}{\log \tau} \log \left(\frac{C_{l} s^{(1-\alpha) / 2} \tau^{(1-\alpha) / 2}}{C_{u} s^{(1-\alpha) / 2}+2}\right) \geq \frac{1-\alpha}{2} .
$$

A similar reasoning leads to

$$
\sigma_{1} \leq \frac{1-\alpha}{2}
$$

Altogether, we get the desired conclusion.

Combining Lemma 4.1 with [13, Theorem 2.1], we are now in a position to establish Theorem 1.1.

Proof of Theorem 1.1, 1) We first derive the second asserted identity

$$
\left(\mathrm{PH}^{1}(D), \mathrm{PH}^{2}(D) \oplus V\right)_{\theta_{2}, 2}=\mathrm{PH}^{1+\theta_{2}}(D), \quad \theta_{2} \in(0, \alpha) .
$$

Let $s \in[0, \alpha)$. Applying [13, Theorem 2.1] together with Lemma 4.1, we obtain the identities

$$
\begin{aligned}
\left(\mathrm{PH}^{2}(D) \oplus V, \mathrm{~L}^{2}(D)\right)_{(1-s) / 2,2} & =\left(\mathrm{PH}^{2}(D), \mathrm{L}^{2}(D)\right)_{(1-s) / 2,2} \\
& =\left(\mathrm{L}^{2}(D), \mathrm{PH}^{2}(D)\right)_{(1+s) / 2,2}=\mathrm{PH}^{1+s}(D) .
\end{aligned}
$$

Choosing $s=0$ yields

$$
\left(\mathrm{PH}^{2}(D) \oplus V, \mathrm{~L}^{2}(D)\right)_{1 / 2,2}=\mathrm{PH}^{1}(D) .
$$


Put now $s=\theta_{2} \in(0, \alpha)$ in $19 p$. Using additionally reiteration interpolation (see [19, Corollary 1.24] for instance), we infer the second desired formula

$$
\begin{aligned}
\left(\mathrm{PH}^{1}(D), \mathrm{PH}^{2}(D) \oplus V\right)_{\theta_{2}, 2} & =\left(\mathrm{PH}^{2}(D) \oplus V, \mathrm{PH}^{1}(D)\right)_{1-\theta_{2}, 2} \\
& =\left(\mathrm{PH}^{2}(D) \oplus V,\left(\mathrm{PH}^{2}(D) \oplus V, \mathrm{~L}^{2}(D)\right)_{1 / 2,2}\right)_{1-\theta_{2}, 2} \\
& =\left(\mathrm{PH}^{2}(D) \oplus V, \mathrm{~L}^{2}(D)\right)_{\left(1-\theta_{2}\right) / 2,2}=\mathrm{PH}^{1+\theta_{2}}(D) .
\end{aligned}
$$

2) Let $\theta_{1} \in(\alpha, 1)$. By [13, Theorem 2.1] and Lemma 4.1, the interpolation space

$$
\left(\mathrm{PH}^{2}(D), \mathrm{L}^{2}(D)\right)_{\left(1-\theta_{1}\right) / 2,2}=\left(\mathrm{L}^{2}(D), \mathrm{PH}^{2}(D)\right)_{\left(1+\theta_{1}\right) / 2,2}=\mathrm{PH}^{1+\theta_{1}}(D)
$$

is a closed subspace of codimension 1 in the space

$$
\begin{aligned}
\left(\mathrm{PH}^{2}(D) \oplus V, \mathrm{~L}^{2}(D)\right)_{\left(1-\theta_{1}\right) / 2,2} \\
=\left(\mathrm{PH}^{2}(D) \oplus V,\left(\mathrm{PH}^{2}(D) \oplus V, \mathrm{~L}^{2}(D)\right)_{1 / 2,2}\right)_{1-\theta_{1}, 2} \\
=\left(\mathrm{PH}^{2}(D) \oplus V, \mathrm{PH}^{1}(D)\right)_{1-\theta_{1}, 2} \\
=\left(\mathrm{PH}^{1}(D), \mathrm{PH}^{2}(D) \oplus V\right)_{\theta_{1}, 2} .
\end{aligned}
$$

Note that here we employ 20 in the second identity. Furthermore, the one-dimensional space $V$ from $(2)$ is also a closed subspace of $\left(\mathrm{PH}^{1}(D), \mathrm{PH}^{2}(D) \oplus V\right)_{\theta_{1}, 2}$ with $V \cap \mathrm{PH}^{1+\theta_{1}}(D)=\{0\}$ (see Corollary 3.3 or 44 for instance). Altogether, we arrive at the first asserted identity

$$
\left(\mathrm{PH}^{1}(D), \mathrm{PH}^{2}(D) \oplus V\right)_{\theta_{1}, 2}=\mathrm{PH}^{1+\theta_{1}}(D) \oplus V .
$$

3) It remains to establish that the space $\operatorname{PH}^{1+\alpha}(D)$ is not closed in $\left(\mathrm{PH}^{1}(D), \mathrm{PH}^{2}(D) \oplus V\right)_{\alpha, 2}$. This is a consequence of the formula

$$
\left(\mathrm{PH}^{1}(D), \mathrm{PH}^{2}(D) \oplus V\right)_{\alpha, 2}=\left(\mathrm{PH}^{2}(D) \oplus V, \mathrm{~L}^{2}(D)\right)_{(1-\alpha) / 2,2},
$$

[13, Theorem 2.1], Lemma 4.1, and the relation

$$
\mathrm{PH}^{1+\alpha}(D)=\left(\mathrm{PH}^{2}(D), \mathrm{L}^{2}(D)\right)_{(1-\alpha) / 2,2} \text {. }
$$

(Note that 22) is verified in the same way as (21).)

Acknowledgements. I want to thank Roland Schnaubelt, Peer Kunstmann, Marlis Hochbruck, Dorothee Frey, and Nick Lindemulder for valuable discussions and advice regarding this paper and my ongoing research. Moreover, I thank Roland Schnaubelt for his careful reading of the manuscript. I furthermore want to express my appreciation for the helpful comments of the anonymous reviewer. The recommendations led to an improvement of the paper.

This research was funded by the Deutsche Forschungsgemeinschaft (DFG, German Research Foundation) - Project-ID 258734477 - SFB 1173. 


\section{References}

[1] I. Asekritova, F. Cobos and N. Kruglyak, Interpolation of closed subspaces and invertibility of operators, Z. Anal. Anwend. 34 (2015), 1-15.

[2] F. Assous, P. Ciarlet Jr. and J. Segré, Numerical solution to the time-dependent Maxwell equations in two-dimensional singular domains: The singular complement method, J. Comput. Phys. 161 (2000), 218-249.

[3] I. Babuška and B. Q. Guo, Regularity of the solution of elliptic problems with piecewise analytic data. Part I. Boundary value problems for linear elliptic equation of second order, SIAM J. Math. Anal. 19 (1988), 172-203.

[4] I. Babuška, B. Andersson, B. Guo, J. M. Melenk and H. S. Oh, Finite element method for solving problems with singular solutions, J. Comput. Appl. Math. 74 (1996), 51-70.

[5] I. Babuška and B. Q. Guo, Optimal estimates for lower and upper bounds of approximation errors in the p-version of the finite element method in two dimensions, Numer. Math. 85 (2000), 219-255.

[6] I. Babuška and B. Q. Guo, Direct and inverse approximation theorems for the pversion of the finite element method in the framework of weighted Besov spaces. Part I. Approximability of functions in the weighted Besov spaces, SIAM J. Numer. Anal. 39 (2001/02), 1512-1538.

[7] P. Ciarlet Jr., F. Lefèvre, S. Lohrengel and S. Nicaise, Weighted regularization for composite materials in electromagnetism, Math. Model. Numer. Anal. 44 (2010), 75-108.

[8] M. Costabel and M. Dauge, Singularities of electromagnetic fields in polyhedral domains, Arch. Ration. Mech. Anal. 151 (2000), 221-276.

[9] M. Costabel, M. Dauge and S. Nicaise, Singularities of Maxwell interface problems, Math. Model. Numer. Anal. 33 (1999), 627-649.

[10] M. Dauge, Elliptic Boundary Value Problems on Corner Domains: Smoothness and Asymptotics of Solutions, Lecture Notes in Math. 1341, Springer, Berlin, 1988.

[11] R. A. DeVore and R. C. Sharpley, Besov spaces and domains in $\mathbb{R}^{d}$, Trans. Amer. Math. Soc. 335 (1993), 843-864.

[12] P. Grisvard, Elliptic Problems in Nonsmooth Domains, Pitman, Boston, 1985.

[13] S. Ivanov and N. Kalton, Interpolation of subspaces and applications to exponential bases, St. Petersburg Math. J. 13 (2002), 221-239.

[14] H. Johnen and K. Scherer, On the equivalence of the $K$-functional and moduli of continuity and some applications, in: Constructive Theory of Functions of Several Variables, W. Schempp and K. Zeller (eds.), Lecture Notes in Math. 571, Springer, Berlin, 1977, 119-140.

[15] A. Jonsson and H. Wallin, A Whitney extension theorem in $L^{p}$ and Besov spaces, Ann. Inst. Fourier (Grenoble) 28 (1978), no. 1, 139-192.

[16] R. B. Kellogg, Singularities in interface problems, in: Numerical Solution of Partial Differential Equations-II. SYNSPADE 1970, B. Hubbard (ed.), Academic Press, New York, 1971, 351-400.

[17] J.-L. Lions and E. Magenes, Non-Homogeneous Boundary Value Problems and Applications, Vol. I, Springer, Berlin, 1972.

[18] J. Löfström, Real interpolation with constraints, J. Approx. Theory 82 (1995), 30-53.

[19] A. Lunardi, Interpolation Theory, 3rd ed., Scuola Normale Superiore, Pisa, 2018.

[20] J. Nečas, Direct Methods in the Theory of Elliptic Equations, 2nd corr., Springer, Berlin, 2012.

[21] S. Nicaise, Polygonal Interface Problems, Lang, Frankfurt am Main, 1993. 
[22] H. Triebel, Allgemeine Legendresche Differentialoperatoren II, Ann. Scuola Norm. Sup. Pisa Cl. Sci. (3) 24 (1970), 1-35.

[23] R. Wallstén, Remarks on interpolation of subspaces, in: Function Spaces and Applications, M. Cwikel et al. (eds.), Lecture Notes in Math. 1302, Springer, Berlin, 1988, $410-419$.

Konstantin Zerulla

Department of Mathematics

Karlsruhe Institute of Technology

Englerstr. 2

76131 Karlsruhe, Germany

E-mail: konstantin.zerulla@kit.edu 